\title{
Use of Fuzzy Matrices for the Diagnosis of Diabetes, Anaemia and Hypertension
}

\author{
Rajeev Gajanan Sapre ${ }^{1}$, Muktai Milind Desai ${ }^{2}$ and Mugdha Mahesh Pokharanakar ${ }^{3}$ \\ ${ }^{1}$ Department of Mathematics, Gogate-Jogalekar College \\ Ratnagiri, Maharashtra, India \\ Email: rgsapre [AT] gmail.com \\ ${ }^{2}$ Department of Mathematics, Gogate-Jogalekar College \\ Ratnagiri, Maharashtra, India \\ Email: muktaidesai [AT] gmail.com \\ ${ }^{3}$ Department of Mathematics, Gogate-Jogalekar College \\ Ratnagiri, Maharashtra, India \\ Email: mmpokharanakar [AT] gmail.com
}

\begin{abstract}
The mathematical model presented here aims to enhance the precision in diagnostic process of diabetes, anaemia and hypertension by means of fuzzy interface. In real life, the imprecise nature of medical documentation and uncertain information provided by patients often do not give the desired degree of confidence to the diagnosis. To that end using the capability of fuzzy logic in representing, interpreting and utilizing data and information that are vague and lack certainty, a new algorithm based on different fuzzy matrices and fuzzy relations is developed. In the process a medical knowledge base is developed with the help of 51 doctors. The model achieved $94.44 \%$ accuracy in the diagnosis, which shows its usefulness. To implement this model-based diagnosis procedure a user-friendly Excel program is designed.
\end{abstract}

Keywords- Fuzzy logic, fuzzy matrices, max-min product, Chi-square test.

\section{INTRODUCTION}

The idea of fuzzy logic as a scientific concept was first used by the computer scientist Zadeh [12]. Medicine is one field in which the applicability of fuzzy logic was recognised quite early in mid 1970s. Within this field, it is the uncertainty found in the process of diagnosis of disease that has most frequently been the focus of application of fuzzy set theory. Imprecise nature of medical documentation and uncertain information supplied by patients make complications in diagnosis of diseases. The best and most useful descriptions of symptoms of diseases often use linguistic terms that are invariably vague [4].

Fuzzy mathematics is a branch of mathematics which captures uncertainty in real life and tries to extract precise meaning from the given vague information. It is a powerful tool for decision-making systems. The fuzzy set framework has been utilized in several ways for modelling medical diagnosis processes. The basic idea in a medical diagnosis is to relate symptoms or patient's signs with possible diseases according to an expert's medical knowledge. In the approach adopted by Sanchez [6], the physician's medical knowledge is represented as a fuzzy relation between symptoms and diseases. Adlassing [1] introduced fuzzy relation model for diagnostic process. Meenakshi and Kaliraja [5] have extended Sanchez's approach for medical diagnosis using the representation of an intervalvalued fuzzy matrix. Elizabeth and Sujatha [2] have developed Sanchez's approach of medical diagnosis using the representation of triangular fuzzy membership matrix. Saravanan and Prasanna [7] presented the application of fuzzy matrices in medicine in which they have used the concept network and concept matrices. Raich et al. [8] established the application of fuzzy matrix in the study of diabetes.

In this paper, we have used fuzzy logic and fuzzy matrices in order to make medical diagnosis more precise. It incorporates the knowledge and experience of 51 physicians and based on that information this mathematical model arrives at the diagnostic conclusions. We have established the distinguishing way to check indication relations for the diagnosis which can be illustrated as 'non-symptom indication - non-occurrence indication - conformability indication'. The comparison of the diagnostic conclusion drawn from our model with actual diagnosis made by doctors gives $94.44 \%$ compatibility and thus establishes a fairly good accuracy of our model. 


\section{RESEARCH METHOD}

This section describes the architecture of the proposed mathematical model which consists of:

\subsection{Formulation of Medical Knowledge Base:}

The physicians' knowledge is represented as a fuzzy relation between symptoms and diseases. Two types of fuzzy relations are defined between symptoms and diseases:

1) An occurrence relation $R_{o}$ :

It provides knowledge about the tendency or frequency of appearance of a symptom when the specific disease is present, i.e. how often does the symptom $s$ occur with disease $d$.

2) A conformability relation $R_{c}$ :

It describes the discriminating power of the symptoms to confirm the presence of the disease, i.e. how strongly does the symptom $s$ confirm disease $d$.

The distinction between occurrence and conformability is useful because though the symptom common to several diseases is present, its occurrence and conformability are different in different diseases.

The above relations are determined from expert medical documentation.

\subsection{Fuzzification:}

Membership grades of all the symptoms of the diseases are determined based on intensity or frequency of the symptom observed in patients inflicted by the selected diseases. This step transforms linguistic terms used to describe the symptoms into numbers.

\subsection{Fuzzy interface:}

Let $\mathrm{D}$ be the set of the selected diseases, $\mathrm{S}$ be the set of the symptoms of the diseases and $\mathrm{P}$ be the set of patients to be diagnosed.

A fuzzy relation $R_{s}$ is defined on the set $\mathrm{P} \times \mathrm{S}$ in which membership grades $R_{s}(p, s)$ (where $\mathrm{p} \in \mathrm{P}, \mathrm{s} \in \mathrm{S}$ ) indicate the degree to which the symptom $s$ is present in patient $p$. A fuzzy relation $\mathrm{R}_{\mathrm{o}}$ is defined on the set $\mathrm{S} \times \mathrm{D}$ where $R_{o}(s, d)$ (where $\mathrm{s} \in \mathrm{S}, \mathrm{d} \in \mathrm{D}$ ) indicates the frequency of occurrence of symptom $s$ with disease $d$. A fuzzy relation $R_{c}$ is also defined on the same set, where $R_{c}(p, d)$ corresponds to the degree to which symptom $s$ confirms the presence of disease $d$.

Using relations $R_{s}, R_{o}$ and $R_{c}$, the following four different indication relations defined on set $\mathrm{P} \times \mathrm{D}$ are calculated:

1) Occurrence indication relation $R_{l}=R_{S} \circ R_{o}$

2) Conformability indication relation $R_{2}=R_{s} \circ R_{c}$

3) Non-occurrence indication relation $R_{3}=R_{S} \circ\left(1-R_{o}\right)$

4) Non-symptom indication relation $R_{4}=\left(1-R_{s}\right) \circ R_{o}$.

For composition of fuzzy relations, max-min product of fuzzy matrices is used.

For an illustration, let $D=\left\{d_{1}, d_{2}\right\}, S=\left\{s_{1}, s_{2}, s_{3}\right\}, P=\left\{p_{1}, p_{2}\right\}$

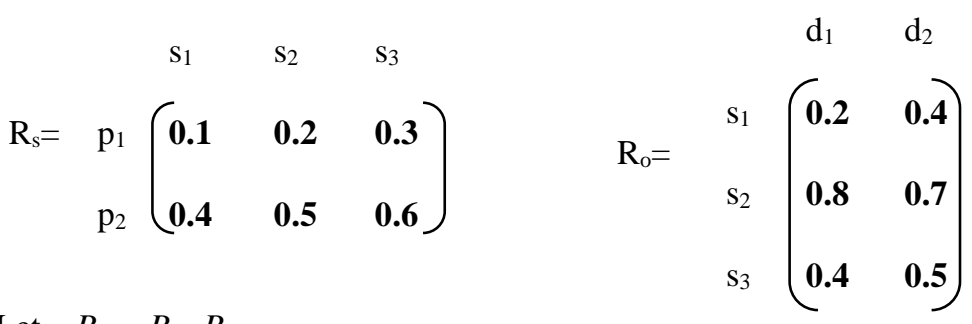

Let $\quad R_{l}=R_{s} \circ R_{o}$

So for every $\mathrm{i}=1,2$ and $\mathrm{j}=1,2, \mathrm{R}_{1}\left(\mathrm{p}_{\mathrm{i}}, \mathrm{d}_{\mathrm{j}}\right)=\max _{\mathrm{s} \in \mathrm{S}}\left\{\min \left\{\mathrm{R}_{\mathrm{s}}\left(\mathrm{p}_{\mathrm{i}}, \mathrm{s}\right), \mathrm{R}_{\mathrm{o}}\left(\mathrm{s}, \mathrm{d}_{\mathrm{j}}\right)\right\}\right\}$

For example, $\mathrm{R}_{1}\left(\mathrm{p}_{1}, \mathrm{~d}_{1}\right)=\max \{\min \{0.1,0.2\}, \min \{0.2,0.8\}, \min \{0.3,0.4\}\}=\max \{0.1,0.2,0.3\}=0.3$.

Similarly, we can calculate $R_{l}\left(p_{i}, d_{j}\right)$ for every $i$ and $j$. 


$$
\mathrm{d}_{1} \quad \mathrm{~d}_{2}
$$

Hence, we get $R_{1=} \quad p_{1}\left(\begin{array}{ll}0.3 & 0.3 \\ & p_{2}\end{array}\right]$

Other indication relations are calculated in the same way.

\subsection{Defuzzification:}

To draw diagnostic conclusions from the above four indication relations, the following rules are developed:

1) Make an excluded diagnosis for a disease $d$ in patient $p$, if $\mathrm{R}_{4}(\mathrm{p}, \mathrm{d}) \geq 0.80$.

2) If for any patient $p$ and any disease $d, \mathrm{R}_{4}(\mathrm{p}, \mathrm{d})<0.80$, find mean of $\mathrm{R}_{3}(\mathrm{p}, \mathrm{d})$ and $\left(1-\mathrm{R}_{1}(\mathrm{p}, \mathrm{d})\right)$, denoted by $m$. Make an excluded diagnosis for a disease $d$ in patient $p$, if $\mathrm{m} \geq 0.80$.

3) If $m<0.80$, make diagnosis as follows:

If $0 \leq \mathrm{R}_{2}(\mathrm{p}, \mathrm{d})<0.30$ - Nil (The disease $d$ is not present in patient $p$.)

If $0.30 \leq \mathrm{R}_{2}(\mathrm{p}, \mathrm{d})<0.40$ - Slight (The disease $d$ is slightly present in patient $p$.)

If $0.40 \leq \mathrm{R}_{2}(\mathrm{p}, \mathrm{d})<0.60$ - Moderate (The disease $d$ is moderately present in patient $p$.)

If $0.60 \leq \mathrm{R}_{2}(\mathrm{p}, \mathrm{d})<0.80$ - High (The disease $d$ is highly present in patient $p$.)

If $0.80 \leq \mathrm{R}_{2}(\mathrm{p}, \mathrm{d}) \leq 1$ - Severe (The disease $d$ is severely present in patient $p$.)

These conclusions are compared with doctors' diagnosis and accuracy of the model is checked. Chi- square test is used as a statistical support for this.

Excel program is developed in order to do above mentioned calculations and make the model user-friendly.

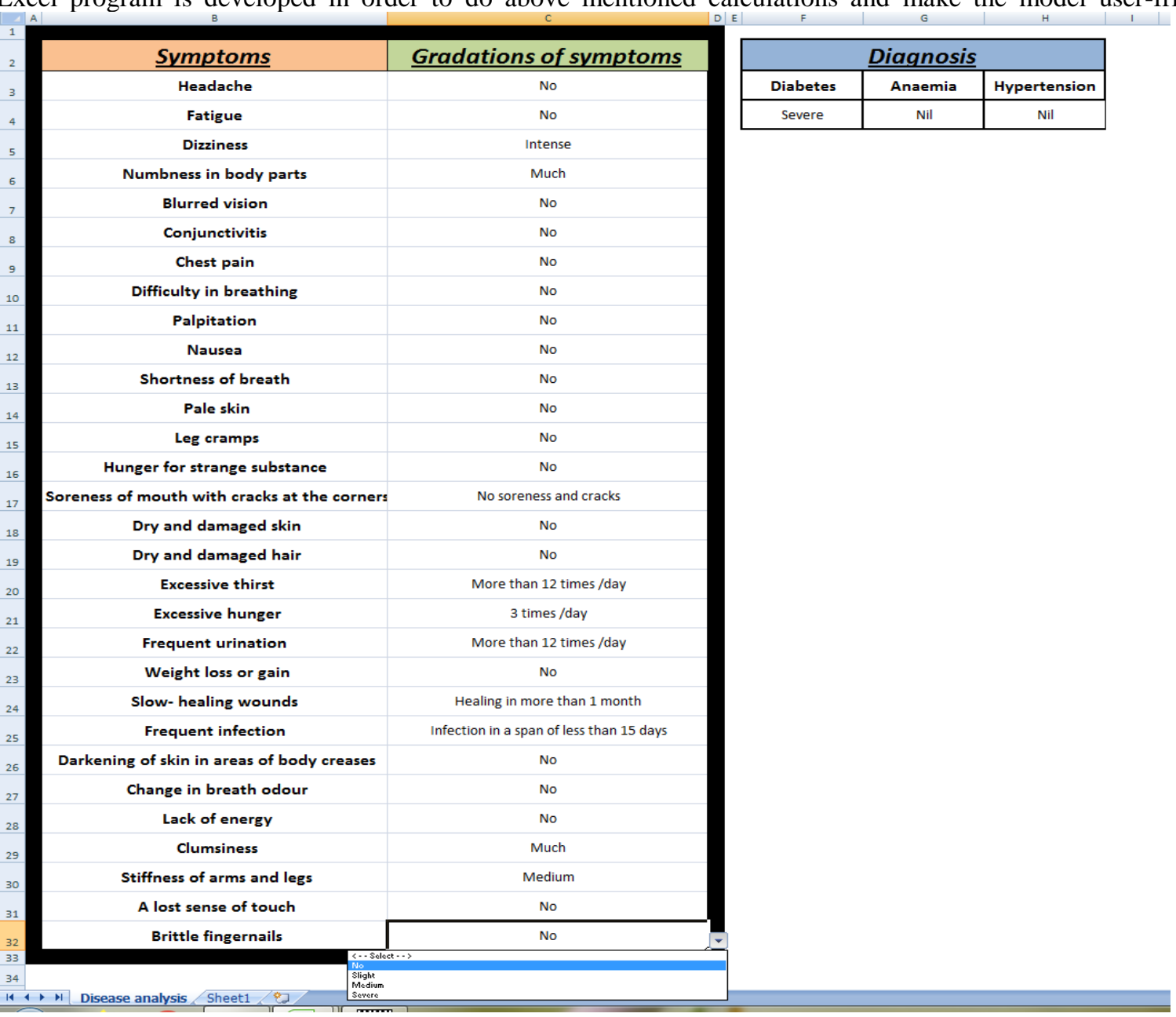

Figure 1: Excel program designed to make the diagnosis 
The Excel program consists of two separate tables as shown in the image above (Figure 1). First table (on the left hand side) contains two columns, viz. symptoms and gradations of symptoms. Second table (on the right hand side) is an indicator of diagnostic conclusions for the three diseases. The first table is used to input the intensity of the user's symptoms and the second table simply gives diagnostic conclusions based on the inputs taken from the first table.

Each cell in the column of gradations of symptoms is provided with a drop-down menu. A user can access the drop-down menu merely by clicking the arrow on the right of the corresponding cell. The drop-down menu consists of the gradations of the symptom based on frequency and intensity of the symptom (like slight, severe etc.) observed in the patients. A user is supposed to opt for a gradation which is observed for that particular symptom. Similarly a user can select gradations for all the thirty symptoms. When a user inputs his gradations of symptom, the diagnostic results for the three diseases can be immediately seen in the second table. The diagnostic results are in the form of linguistic terms like nil, slight, moderate etc. which are easy to interpret.

\section{PROCEDURE EMPLOYED}

Here we have selected three diseases viz., Diabetes, Anaemia and Hypertension as a case study. The reason for choice of these diseases is that they are the most prevalent diseases in India as we can see from WHO (World Health Organization) reports. In India, according to WHO reports in the year 2016, the prevalence of diabetes in adults is $7.8 \%$ [9], prevalence of anaemia among women of reproductive age is 51.43\% [10] and according to WHO report in the year 2018, prevalence of hypertension in adults is $24 \%$ [11].

After selecting the three diseases, we listed 30 symptoms of these diseases which are commonly observed in patients suffering by them.

Then we developed medical knowledge base with the help of the doctors. For the formulation of the knowledge base, we considered occurrence of a symptom in corresponding disease and confirmation of a disease from corresponding symptom. Occurrence of a symptom in corresponding disease explains tendency or frequency of appearance of symptom when the specific disease is present; it corresponds to the question, 'how often does symptom $s$ occur with disease $d$ ?'. Confirmation of a disease from corresponding symptom describes the discriminating power of the symptom to confirm the presence of the disease; it corresponds to the question, 'how strongly does symptom $s$ confirm disease $d$ ?'. We provided blank charts (Appendix) to randomly selected 51 doctors in Ratnagiri district to develop a knowledge base consisting of occurrence of a symptom in corresponding disease and the confirmation of a disease from particular symptom. For occurrence of a symptom, we asked the doctors to write the number of patients in which that particular symptom is observed out of 100 patients of the corresponding disease. For instance, if a doctor has examined 100 patients of diabetes and if he/she finds 'excessive thirst' in 90 patients out of 100, he/she has to write 90 as occurrence of excessive thirst in diabetes. For confirmation of a disease, we asked the doctors to write the percentage by which he/she confirms the presence of that particular disease when he/she observes the corresponding symptom in the patient. E.g., if a doctor observes 'pale skin' of a patient and if he/she thinks that the possibility that the patient would be suffering from anaemia is $80 \%$, then he/she has to write 80 as confirmation of anaemia from pale skin. We divide these numbers by 100 so that they lie in $[0,1]$.

We calculated mean of the data collected to find central tendency of the doctors' data as shown in table 1 .

For fuzzification, we assigned membership grades to each of the 30 symptoms based on intensity or frequency of the symptom observed in a patient. The grades are as follows:

1) Headache:

Continuous severe: $1.00 \quad$ Continuous mild: 0.75

Daily: $0.50 \quad$ Occasionally: 0.25

No: 0.00

2) Fatigue:

Very much: 1.00

Slight: 0.33

Medium: 0.66

No: 0.00

3) Dizziness:

Intense: 1.00

Medium: 0.66

Slight: 0.33

No: 0.00

4) Numbness in body parts:

Much: 1.00

Medium: 0.66

Vey less: 0.33

No: 0.00

5) Blurred vision:

High: 1.00

Medium: 0.66

Very slight: 0.33

No: 0.00

Table 1: Mean of knowledge-base obtained from experts 


\begin{tabular}{|c|c|c|c|c|c|c|c|}
\hline \multirow[t]{2}{*}{$\begin{array}{l}\text { Sr. } \\
\text { No. }\end{array}$} & \multirow[t]{2}{*}{ SYMPTOMS } & \multicolumn{2}{|c|}{ DIABETES } & \multicolumn{2}{|c|}{ ANAEMIA } & \multicolumn{2}{|c|}{ HYPERTENSION } \\
\hline & & $\mathbf{O}$ & $\mathrm{C}$ & $\mathbf{O}$ & $\mathrm{C}$ & $\mathbf{O}$ & $\mathbf{C}$ \\
\hline 1. & Headache & 0.02 & 0.02 & 0.49 & 0.37 & 0.8 & 0.7 \\
\hline 2. & Fatigue & 0.71 & 0.64 & 0.85 & 0.75 & 0.33 & 0.22 \\
\hline 3. & Dizziness & 0.39 & 0.36 & 0.66 & 0.59 & 0.58 & 0.46 \\
\hline 4. & Numbness in body parts & 0.65 & 0.54 & 0.32 & 0.27 & 0.35 & 0.24 \\
\hline 5 . & Blurred vision & 0.52 & 0.45 & 0.25 & 0.19 & 0.37 & 0.27 \\
\hline 6. & Conjunctivitis & 0.28 & 0.12 & 0.04 & 0.04 & 0.04 & 0.03 \\
\hline 7. & Chest pain & 0.15 & 0.12 & 0.13 & 0.11 & 0.75 & 0.65 \\
\hline 8. & Difficulty in breathing & 0.16 & 0.07 & 0.5 & 0.37 & 0.51 & 0.41 \\
\hline 9. & Palpitation & 0.18 & 0.07 & 0.47 & 0.41 & 0.63 & 0.49 \\
\hline 10. & Nausea & 0.14 & 0.11 & 0.31 & 0.18 & 0.38 & 0.28 \\
\hline 11. & Shortness of breath & 0.16 & 0.12 & 0.6 & 0.52 & 0.52 & 0.49 \\
\hline 12. & Pale skin & 0.2 & 0.1 & 0.89 & 0.8 & 0.09 & 0.05 \\
\hline 13. & Leg cramps & 0.48 & 0.41 & 0.6 & 0.56 & 0.21 & 0.11 \\
\hline 14. & Hunger for strange substance & 0.15 & 0.09 & 0.37 & 0.24 & 0.02 & 0.01 \\
\hline 15. & Soreness of mouth with cracks at the corners & 0.15 & 0.05 & 0.55 & 0.54 & 0.04 & 0.02 \\
\hline 16. & Dry and damaged skin & 0.25 & 0.2 & 0.53 & 0.4 & 0.04 & 0.02 \\
\hline 17. & Dry and damaged hair & 0.17 & 0.08 & 0.52 & 0.43 & 0.03 & 0.02 \\
\hline 18. & Excessive thirst & 0.85 & 0.81 & 0.29 & 0.18 & 0.08 & 0.03 \\
\hline 19. & Excessive hunger & 0.86 & 0.74 & 0.08 & 0.05 & 0.05 & 0.02 \\
\hline 20. & Frequent urination & 0.87 & 0.88 & 0.16 & 0.1 & 0.15 & 0.09 \\
\hline 21. & Weight loss or gain & 0.79 & 0.66 & 0.6 & 0.54 & 0.13 & 0.06 \\
\hline 22. & Slow- healing wounds & 0.79 & 0.84 & 0.25 & 0.15 & 0.07 & 0.02 \\
\hline 23. & Frequent infection & 0.77 & 0.81 & 0.3 & 0.23 & 0.09 & 0.09 \\
\hline 24. & Darkening of skin in areas of body creases & 0.29 & 0.23 & 0.22 & 0.14 & 0.09 & 0.04 \\
\hline 25. & Change in breath odour & 0.47 & 0.36 & 0.17 & 0.11 & 0.07 & 0.03 \\
\hline 26. & Lack of energy & 0.66 & 0.6 & 0.83 & 0.73 & 0.32 & 0.23 \\
\hline 27. & Clumsiness & 0.26 & 0.15 & 0.39 & 0.32 & 0.23 & 0.08 \\
\hline 28. & Stiffness of arms and legs & 0.19 & 0.15 & 0.39 & 0.27 & 0.04 & 0.03 \\
\hline 29. & A lost sense of touch & 0.39 & 0.38 & 0.16 & 0.11 & 0.08 & 0.09 \\
\hline 30. & Brittle fingernails & 0.34 & 0.24 & 0.61 & 0.54 & 0.1 & 0.07 \\
\hline
\end{tabular}

6) Conjunctivitis:

Vision affecting: 1.00

Insignificant: 0.33

7) Chest pain:

Severe and intolerable: 1.00

Moderate: 0.50

8) Difficulty in breathing:

Very much: 1.00

Slight: 0.33

9) Palpitation:

Drastic: 1.00

Slight: 0.33

10) Nausea:

Extreme: 1.00

Mild: 0.33

11) Shortness of breath:

High: 1.00

Slight: 0.33

12) Pale skin:

Very much: 1.00

Slight: 0.33

13) Leg cramps:

2-3 times /day: 1.00

1-3 times /week: 0.50
Medium: 0.66

No: 0.00

High but tolerable: 0.75

Slight: 0.25

No: 0.00

Medium: 0.66

No: 0.00

Medium: 0.66

No: 0.00

Medium: 0.66

No: 0.00

Medium: 0.66

No: 0.00

Medium: 0.66

No: 0.00

4-13 times /week: 0.75

Occasionally: 0.25

No: 0.00 
14) Hunger for strange substance:

Adversely health affecting: 1.00

Medium: 0.66

Slight: 0.33

No: 0.00

15) Soreness of mouth with cracks at the corners:

This lasts for-

More than 14 days: 1.00

7-14 days: 0.75

3-6 days: 0.50

Less than 3 days: 0.25

No soreness and cracks: 0.00

16) Dry and damaged skin:

Cracked skin: 1.00

Severe: 0.75

Medium: 0.50

Slight: 0.25

No: 0.00

17) Dry and damaged hair:

Brittle hair: 1.00

Severe: 0.75

Medium: 0.50

Slight: 0.25

No: 0.00

18) Excessive thirst:

More than 12 times /day: 1.00

8-12 times /day: 0.75

6-7 times /day: 0.50

4-5 times /day: 0.25

Less than 4 times /day: 0.00

19) Excessive hunger:

More than 5 times /day: 1.00

5 times /day: 0.75

4 times /day: 0.50

3 times /day: 0.25

Less than 3 times /day: 0.00

20) Frequent urination:

More than 12 times /day: 1.00

9-12 times /day: 0.75

6-8 times /day: 0.50

4-5 times /day: 0.25

Less than 4 times /day: 0.00

21) Weight loss/gain:

Extreme: 1.00

High: 0.75

Moderate: 0.50

Slight: 0.25

No: 0.00

22) Slow-healing wounds:

Healing in more than 1 month: 1.00 Healing in 1 month: 0.75

Healing in 16-30 days: 0.50

Healing in 8-15 days: 0.25

Healing in less than 8 days: 0.00

23) Frequent infection:

Infection in a span of-

Less than 15 days: 1.00

15 days- 1 month: 0.75

2 months- 3 months: 0.25

More than 3 months: 0.00

24) Darkening of skin in areas of body creases:

Severe: 1.00

Medium: 0.50

25) Change in breath odour:

Strong: 1.00

Slight: 0.33

26) Lack of energy:

Much: 1.00

Slight: 0.33

27) Clumsiness:

Much: 1.00

Slight: 0.33

28) Stiffness of arms and legs:

Severe: 1.00

Slight: 0.33

Very much: 0.75

Slight: 0.25

No: 0.00
Medium: 0.66

No: 0.00

More: 0.66

No: 0.00

More: 0.66

No: 0.00

Medium: 0.66

No: 0.00

Medium: 0.66

No: 0.00

Medium: 0.66

No: 0.00

Severe: 1.00

Slight: 0.33

30) Brittle fingernails:

Severe: 1.00

Slight: 0.33

In this case, $\mathrm{D}=\{$ Diabetes, Anaemia, Hypertension $\}, \mathrm{S}=$ set of 30 symptoms $=\left\{\mathrm{s}_{1}, \mathrm{~s}_{2}, \ldots, \mathrm{s}_{30}\right\}$.

For illustration of our methodology of diagnosis, we are taking example of three dummy patients here. So, $\mathrm{P}=\left\{\mathrm{p}_{1}, \mathrm{p}_{2}, \mathrm{p}_{3}\right\}$.

By considering intensity or frequency of symptoms observed in these three patients, we have defined a fuzzy relation $R_{s}$ on the set $\mathrm{P} \times \mathrm{S}$ in which membership grades $R_{s}(p, s)$ (where $\mathrm{p} \in \mathrm{P}, \mathrm{s} \in \mathrm{S}$ ) indicate the degree to which the symptom $s$ is present in patient $p$ as follows: 
By considering the knowledge base, we defined fuzzy relations $R_{o}$ and $R_{c}$ on the set $\mathrm{S} \times \mathrm{D}$ where $R_{o}(s, d)(\mathrm{s} \in \mathrm{S}$, $\mathrm{d} \in \mathrm{D}$ ) indicates the frequency of occurrence of symptom $s$ with disease $d$ and $R_{c}(p, d)$ corresponds to the degree to which symptom $s$ confirms the presence of disease $d$ as follows:

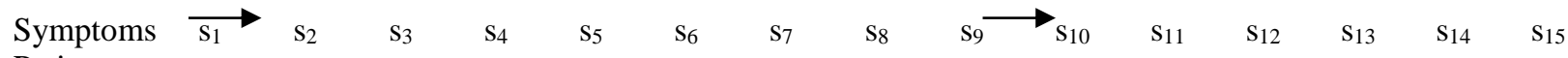
Patients

\begin{tabular}{|c|c|c|c|c|c|c|c|c|c|c|c|c|c|c|c|}
\hline p1 & 0.75 & 0.33 & 0.66 & 1.00 & 0.33 & 0.00 & 0.50 & 0.33 & 0.66 & 0.66 & 0.33 & 0.00 & 0.25 & 0.00 & 0.00 \\
\hline 0 & 0.00 & 0.66 & 0.66 & 1.00 & 0.33 & 0.00 & 0.00 & 0.00 & 0.00 & 0.00 & 0.00 & 0.00 & 0.50 & 0.00 & \\
\hline & 0.25 & 1.00 & 0.66 & 0.66 & 0.00 & 0.00 & 0.25 & 0.33 & 0.33 & $\mathbf{0 . 3 3}$ & 0.66 & 0.66 & 0.75 & 0.33 & \\
\hline
\end{tabular}

$\mathrm{R}_{\mathrm{o}}$ : Diseases

$$
\begin{array}{lllllllllllllll}
\mathrm{s}_{16} & \mathrm{~s}_{17} & \mathrm{~s}_{18} & \mathrm{~s}_{19} & \mathrm{~s}_{20} & \mathrm{~s}_{21} & \mathrm{~s}_{22} & \mathrm{~s}_{23} & \mathrm{~s}_{24} & \mathrm{~s}_{25} & \mathrm{~s}_{26} & \mathrm{~s}_{27} & \mathrm{~s}_{28} & \mathrm{~s}_{29} & \mathrm{~s}_{30} \\
\mathbf{0 . 0 0} & \mathbf{0 . 0 0} & \mathbf{0 . 0 0} & \mathbf{0 . 0 0} & \mathbf{0 . 2 5} & \mathbf{0 . 0 0} & \mathbf{0 . 0 0} & \mathbf{0 . 0 0} & \mathbf{0 . 0 0} & \mathbf{0 . 0 0} & \mathbf{0 . 3 3} & \mathbf{0 . 6 6} & \mathbf{0 . 3 3} & \mathbf{0 . 3 3} & \mathbf{0 . 0 0} \\
\mathbf{0 . 2 5} & \mathbf{0 . 0 0} & \mathbf{0 . 7 5} & \mathbf{0 . 5 0} & \mathbf{0 . 7 5} & \mathbf{0 . 5 0} & \mathbf{0 . 5 0} & \mathbf{0 . 7 5} & \mathbf{0 . 0 0} & \mathbf{0 . 6 6} & \mathbf{0 . 6 6} & \mathbf{0 . 6 6} & \mathbf{0 . 0 0} & \mathbf{0 . 3 3} & \mathbf{0 . 3 3} \\
& & & & & & & & & & & & & & \\
\mathbf{0 . 5 0} & \mathbf{0 . 5 0} & \mathbf{0 . 2 5} & \mathbf{0 . 0 0} & \mathbf{0 . 0 0} & \mathbf{0 . 5 0} & \mathbf{0 . 2 5} & \mathbf{0 . 2 5} & \mathbf{0 . 2 5} & \mathbf{0 . 3 3} & \mathbf{1 . 0 0} & \mathbf{0 . 6 6} & \mathbf{0 . 0 0} & \mathbf{0 . 0 0} & \mathbf{0 . 6 6}
\end{array}
$$

\section{Symptoms}

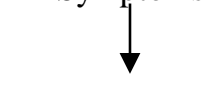

\begin{tabular}{|ll}
\hline & \\
\hline $\mathbf{0 . 0 2}$ & $\mathbf{0 . 4}$ \\
$\mathbf{0 . 7 1}$ & $\mathbf{0 . 8}$ \\
$\mathbf{0 . 3 9}$ & $\mathbf{0 . 6}$ \\
$\mathbf{0 . 6 5}$ & $\mathbf{0 . 3}$ \\
$\mathbf{0 . 5 2}$ & $\mathbf{0 . 2}$ \\
$\mathbf{0 . 2 8}$ & $\mathbf{0 . 0}$ \\
$\mathbf{0 . 1 6}$ & $\mathbf{0 . 1 3}$ \\
$\mathbf{0 . 1 8}$ & $\mathbf{0 . 5}$ \\
$\mathbf{0 . 1 4}$ & $\mathbf{0 . 3}$ \\
$\mathbf{0 . 1 6}$ & $\mathbf{0 . 6}$ \\
$\mathbf{0 . 2}$ & $\mathbf{0 . 8}$ \\
$\mathbf{0 . 4 8}$ & $\mathbf{0 . 6}$ \\
$\mathbf{0 . 1 5}$ & $\mathbf{0 . 3}$ \\
$\mathbf{0 . 1 5}$ & $\mathbf{0 . 5}$ \\
$\mathbf{0 . 2 5}$ & $\mathbf{0 . 5}$ \\
$\mathbf{0 . 1 7}$ & $\mathbf{0 . 5}$ \\
$\mathbf{0 . 8 5}$ & $\mathbf{0 . 2 9}$ \\
$\mathbf{0 . 8 6}$ & $\mathbf{0 . 0 8}$ \\
$\mathbf{0 . 8 7}$ & $\mathbf{0 . 1 6}$ \\
$\mathbf{0 . 7 9}$ & $\mathbf{0 . 6}$ \\
$\mathbf{0 . 7 9}$ & $\mathbf{0 . 2 5}$ \\
$\mathbf{0 . 7 7}$ & $\mathbf{0 . 3}$ \\
$\mathbf{0 . 2 9}$ & $\mathbf{0 . 2 2}$ \\
$\mathbf{0 . 4 7}$ & $\mathbf{0 . 1 7}$ \\
$\mathbf{0 . 6 6}$ & $\mathbf{0 . 8 3}$ \\
$\mathbf{0 . 2 6}$ & $\mathbf{0 . 3 9}$ \\
$\mathbf{0 . 1 9}$ & $\mathbf{0 . 3 9}$ \\
$\mathbf{0 . 3 9}$ & $\mathbf{0 . 1 6}$ \\
$\mathbf{0 . 3 4}$ & $\mathbf{0 . 6 1}$ \\
\hline & \\
\hline
\end{tabular}

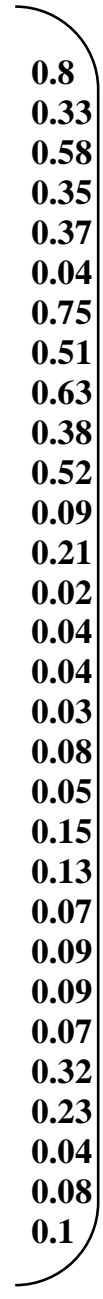

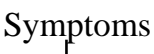

$\mathrm{S}_{1}$

$\mathrm{S}_{2}$

$\mathrm{S}_{3}$

$\mathrm{S}_{4}$

S5

S6

S7

$\mathrm{S} 8$

S9

$\mathrm{S}_{10}$

$\mathrm{S}_{11}$

$\mathrm{S}_{12}$

$\mathrm{S}_{13}$

$\mathrm{S}_{14}$

$\mathrm{S}_{15}$

$\mathrm{S}_{16}$

$\mathrm{S}_{17}$

$\mathrm{S}_{18}$

$\mathrm{S}_{19}$

$\mathrm{S}_{20}$

$\mathrm{S}_{21}$

$\mathrm{S}_{22}$

$\mathrm{S}_{23}$

$\mathrm{S}_{24}$

$\mathrm{S}_{25}$

$\mathrm{S}_{26}$

$\mathrm{S}_{27}$

$\mathrm{S}_{28}$

$\mathrm{S}_{29}$

$\mathrm{S}_{30}$

\begin{tabular}{|l}
\hline 0.02 \\
0.64 \\
0.36 \\
0.54 \\
0.45 \\
0.12 \\
0.12 \\
0.07 \\
0.07 \\
0.11 \\
0.12 \\
0.1 \\
0.41 \\
0.09 \\
0.05 \\
0.2 \\
0.08 \\
0.81 \\
0.74 \\
0.88 \\
0.66 \\
0.84 \\
0.81 \\
0.23 \\
0.36 \\
0.6 \\
0.15 \\
0.15 \\
0.38 \\
0.24 \\
\\
\hline
\end{tabular}

0.75

0.59

0.27

0.19

0.04

0.11

0.37

0.41

0.18

0.52

0.8

0.56

0.24

0.54

0.4

0.43

0.18

0.05

0.1

0.54

0.15

0.23

0.14

0.11

0.73

0.32

0.27

0.11

0.54
0.7

0.22

0.46

0.24

0.27

0.03

0.65

0.41

0.49

0.28

0.49

0.05

0.11

0.01

0.02

0.02

0.02

0.03

0.02

0.09

0.06

0.02

0.09

0.04

0.03

0.23

0.08

0.03

0.09

0.07

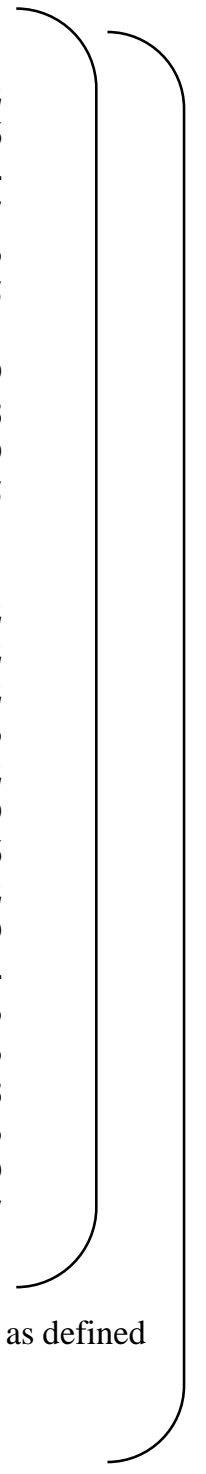

Using these relations $R_{s}, R_{o}$ and $R_{c}$, we calculated four different indication relations viz. $R_{l}, R_{2}, R_{3}, R_{4}$ as defined in section 2.3 above.

The calculated indication relations are as follows:

$\mathrm{R}_{1}=\mathrm{R}_{\mathrm{s}} \circ \mathrm{R}_{\mathrm{o}}$

$\mathrm{R}_{2}=\mathrm{R}_{\mathrm{s}} \circ \mathrm{R}_{\mathrm{c}}$ 


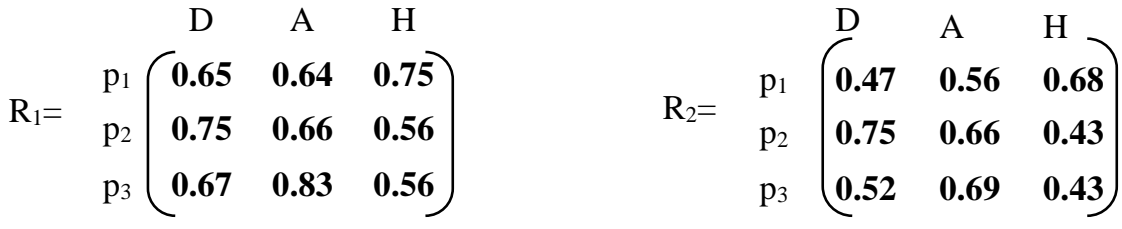

$$
\begin{aligned}
& \mathrm{R}_{3}=\mathrm{R}_{\mathrm{s}} \circ\left(1-\mathrm{R}_{\mathrm{o}}\right) \quad \mathrm{R}_{4}=\left(1-\mathrm{R}_{\mathrm{s}}\right) \circ \mathrm{R}_{\mathrm{o}}
\end{aligned}
$$

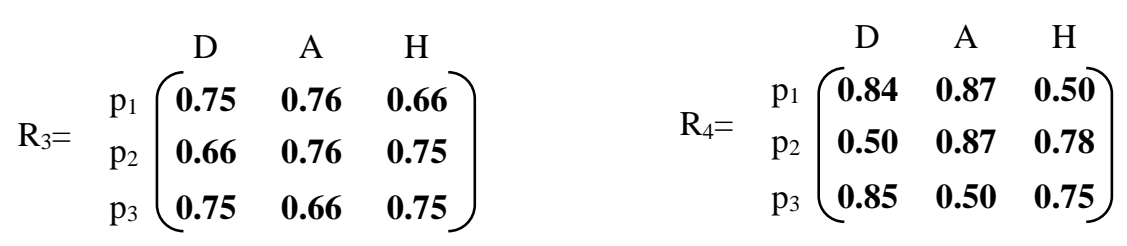

To draw diagnostic conclusions from the above mentioned four indication relations, we developed some rules. The rules are as follows:

1) Make an excluded diagnosis for a disease $d$ in patient $p$ if $R_{4}(p, d) \geq 0.80$.

2) If for any patient $p$ and any disease $d, \mathrm{R}_{4}(\mathrm{p}, \mathrm{d})<0.80$, find mean of $\mathrm{R}_{3}(\mathrm{p}, \mathrm{d})$ and $\left(1-\mathrm{R}_{1}(\mathrm{p}, \mathrm{d})\right)$, denoted by $m$. Make an excluded diagnosis for a disease $d$ in patient $p$ if $\mathrm{m} \geq 0.80$.

3) If $\mathrm{m}<0.80$, make diagnosis as follows:

$$
\begin{aligned}
& \text { If } 0 \leq \mathrm{R}_{2}(\mathrm{p}, \mathrm{d})<0.30-\text { Nil } \\
& \text { If } 0.30 \leq \mathrm{R}_{2}(\mathrm{p}, \mathrm{d})<0.40 \text { - Slight } \\
& \text { If } 0.40 \leq \mathrm{R}_{2}(\mathrm{p}, \mathrm{d})<0.60 \text { - Moderate } \\
& \text { If } 0.60 \leq \mathrm{R}_{2}(\mathrm{p}, \mathrm{d})<0.80 \text { - High } \\
& \text { If } 0.80 \leq \mathrm{R}_{2}(\mathrm{p}, \mathrm{d}) \leq 1-\text { Severe }
\end{aligned}
$$

If we make diagnosis of $\mathrm{p}_{1}, \mathrm{p}_{2}$ and $\mathrm{p}_{3}$ using above rules, we infer that patient $p_{1}$ has High hypertension, patient $p_{2}$ has High diabetes and patient $p_{3}$ has High anaemia and Moderate hypertension.

To perform these calculations, an excel program is designed in which the linguistic description of one's symptoms can be given as input and diagnosis can be obtained as an output.

Using this program, we diagnosed 30 patients by using this model and compared this diagnosis with doctors' diagnosis. Our diagnosis matched perfectly with doctors' diagnosis in case of diabetes and anaemia and in case of hypertension it matched in 25 patients out of 30 patients.

The level of accuracy of the model can be calculated as:

Accuracy $=[(\text { The number of accurate data }) /(\text { The number of total data })]^{* 100}=(85 / 90) * 100=94.44 \%$

We used Chi square test [3] for hypothesis testing as follows:

- For testing the reliability of our mathematical model statistically, we took the help of Chi-square test, popularly known as 'goodness of fit test'. Before applying the Chi-square test, we mainly assumed that the diagnosis made by doctors is $100 \%$ correct.

- Our null hypothesis $H_{0}$ is given as:

$H_{0}$ : There is no difference between the diagnosis of the doctors' and ours.

- Corresponding alternative hypothesis $H_{l}$ is given as:

$H_{l}$ : Our diagnosis is different from doctors'.

- Accepted significance level $(\alpha)=0.05$,

- Degrees of freedom= 2

- $\quad$ Critical value $=5.991$

Table 2: Contingency Table for Chi square test

\begin{tabular}{|l|l|l|}
\hline & Observed & Expected \\
\hline Diabetes & 30 & 30 \\
\hline Anaemia & 30 & 30 \\
\hline Hypertension & 25 & 30 \\
\hline
\end{tabular}


- Formula for calculating Chi-square test is given as:

$\chi^{2}=\Sigma\left[(\mathrm{O}-\mathrm{E})^{2} / \mathrm{E}\right]$ where $\mathrm{O}$ is observed frequency, $\mathrm{E}$ is expected frequency.

- From the contingency table, we calculated chi-square $\left(\chi^{2}\right)$ as $\chi^{2}=\left((30-30)^{2} / 30\right)+\left((30-30)^{2} / 30\right)+\left((25-30)^{2} / 30\right)=0.83$.

- Since $0.83<5.991$ i.e. $\chi^{2}<$ critical value, hence null hypothesis $\mathrm{H}_{0}$ is accepted.

We concluded that there is no difference between the diagnosis of the doctors' and ours.

\section{CONCLUSION}

Fuzzy logic can be efficiently used to make medical diagnosis more affirmatory and precise. The diagnosis made by 'max-min' composition of fuzzy relations formulated by using 'mean' of data collected from the doctors is in accordance with the doctors' diagnosis. The proposed mathematical model has $94.44 \%$ accuracy and chi- square test certifies its reliability statistically. The new algorithm presented in this research paper tries to represent the general idea behind the diagnostic process mathematically. The systematic way to check the indication relations, specifically 'non-symptom indication - non-occurrence indication - conformability indication' is the distinguishing feature of this algorithm.

This is an effort to capture experts' knowledge and make it available to layman and to general practitioners also. Surely this is not a substitute to doctor's diagnosis but it will help a doctor to confirm his/her diagnosis.

Diagnostic conclusions drawn from this mathematical model may be considered as second opinion to the doctor's diagnosis. Altogether this mathematical model aims to maximize the diagnostic accuracy. The excel program is developed to make this model free from tedious calculations and to make it user-friendly.

Incorrect inputs given by a patient will surely lead to incorrect diagnostic conclusions rendering the proposed mathematical model off target which is the main limitation.

Future works include the development of software and an android app after more data collection from doctors. Also, development of new algorithms for the sake of more precision is one of the future goals.

\section{ACKNOWLEDGEMENT}

The authors would like to thank the doctors for their assistance with the formulation of medical knowledge base. The help given by the doctors to check the reliability of diagnostic conclusions drawn by the proposed mathematical model surely deserves the sincere appreciation of the authors. The authors solemnly wish to thank Dr. Vivek Patkar, an Independent scientist for giving his valuable suggestions.

\section{REFERENCES}

[1] Adlassing, K.P., "Fuzzy set theory in medical diagnosis", IEEE Transaction systems Man Cybernetics, 16(2), pp. 260-265, 1986.

[2] Elizabeth, S. and Sujatha, L., "Application of fuzzy membership matrix in medical diagnosis and decision making", Applied Mathematical Sciences, Vol. 7, pp. 6297-6307, 2013.

[3] Gupta, S.P.: Statistical Methods, S. Chand \& sons, New Delhi, 1976.

[4] Klir, G. and Yuan, B., Fuzzy Sets and Fuzzy Logic: Theory and Applications, Prentice- Hall, Upper Saddle River, New Jersey, 1995.

[5] Meenakshi, A. R. and Kaliraja M., "An application of interval-valued fuzzy matrices in medical diagnosis", International Journal of Mathematical Analysis, Vol. 5, pp. 1791-1802, 2011.

[6] Sanchez, E., "Inverse of fuzzy relations, application to possibility distribution and medical diagnosis", Fuzzy Sets and Systems", Vol. 2, pp. 75-86, 1979.

[7] Saravanan, K. and Prasanna, J.S., "Applications of fuzzy matrices in medicine", Global Journal of Pure and Applied Mathematics, Vol.12, number 2, pp. 80-84, 2016.

[8] Raich, V., Tripathi, R.K., Agarwal, V. and Dalal, S., "Application of fuzzy matrix in the study of diabetes", World Academy of Science, Engineering and Technology, Vol. 56, pp. 22-28, 2009.

[9] World Health Organization - Diabetes country profiles. https://www.who.int/diabetes/country-profiles/ind_en.pdf?ua=1 (2016)

[10] World Health Organization - Prevalence of anaemia in women of reproductive age (\%). https://www.who.int/data/gho/data/indicators/indicator-details/GHO/prevalence-of-anaemia-in-women-ofreproductive-age-(-) (2016)

[11] World Health Organization - Noncommunicable Diseases (NCD) Country Profiles. https://www.who.int/nmh/countries/2018/ind_en.pdf?ua=1 (2018) 
[12] Zadeh, L. A., "Fuzzy set", Information and Control, Vol. 8, pp. 338-353, 1965.

\section{APPENDIX}

Table: Blank chart provided to doctors to formulate the knowledge base consisting of occurrence and confirmation

$(\mathrm{O}=$ Occurrence of a symptom, $\mathrm{C}=$ Confirmation of a disease $)$

Date:

Doctor's signature

\begin{tabular}{|c|c|c|c|c|c|c|c|}
\hline $\begin{array}{l}\text { Sr. } \\
\text { No. }\end{array}$ & SYMPTOMS & DI & TES & & & & ГENSION \\
\hline & & $\mathbf{O}$ & $\mathbf{C}$ & $\mathbf{O}$ & $\mathbf{C}$ & $\mathbf{O}$ & $\mathbf{C}$ \\
\hline 1. & Headache & & & & & & \\
\hline 2. & Fatigue & & & & & & \\
\hline 3. & Dizziness & & & & & & \\
\hline 4. & Numbness in body parts & & & & & & \\
\hline 5. & Blurred vision & & & & & & \\
\hline 6. & Conjunctivitis & & & & & & \\
\hline 7. & Chest pain & & & & & & \\
\hline 8. & Difficulty in breathing & & & & & & \\
\hline 9. & Palpitation & & & & & & \\
\hline 10. & Nausea & & & & & & \\
\hline 11. & Shortness of breath & & & & & & \\
\hline 12. & Pale skin & & & & & & \\
\hline 13. & Leg cramps & & & & & & \\
\hline 14. & Hunger for strange substance & & & & & & \\
\hline 15. & Soreness of mouth with cracks at the corners & & & & & & \\
\hline 16. & Dry and damaged skin & & & & & & \\
\hline 17. & Dry and damaged hair & & & & & & \\
\hline 18. & Excessive thirst & & & & & & \\
\hline 19. & Excessive hunger & & & & & & \\
\hline 20. & Frequent urination & & & & & & \\
\hline 21. & Weight loss or gain & & & & & & \\
\hline 22. & Slow- healing wounds & & & & & & \\
\hline 23. & Frequent infection & & & & & & \\
\hline 24. & Darkening of skin in areas of body creases & & & & & & \\
\hline 25. & Change in breath odour & & & & & & \\
\hline 26. & Lack of energy & & & & & & \\
\hline 27. & Clumsiness & & & & & & \\
\hline 28. & Stiffness of arms and legs & & & & & & \\
\hline 29. & A lost sense of touch & & & & & & \\
\hline 30. & Brittle fingernails & & & & & & \\
\hline
\end{tabular}

